\title{
Determinants of Time to Treatment Dropout among Tuberculosis Patients in Buno-Bedele and Illu Ababora Zones, Oromia Regional State, Ethiopia
}

WOLDEMARIAM GOBENA ( $\sim$ woldenegniko@gmail.com )

Mettu University

DEREJE ABABU

Mettu University

AZMERAW GETANEH

Mettu University

\section{Research Article}

Keywords: Tuberculosis, HIV co-infection, Cox-regression

Posted Date: April 30th, 2021

DOI: https://doi.org/10.21203/rs.3.rs-322443/v1

License: (c) (i) This work is licensed under a Creative Commons Attribution 4.0 International License.

Read Full License 


\title{
Determinants of Time to Treatment Dropout among Tuberculosis Patients in Buno-Bedele and Illu Ababora Zones, Oromia Regional State, Ethiopia
}

Woldemariam Erkalo ${ }^{1 *}$ (Email: woldez21@ @gmail.com; ORCID iD: 0000-0002-0215-3436)

Dereje Gebeyehu ${ }^{2}$ (Email: dgebe20@gmail.com)

Azmeraw Misganaw ${ }^{3}$ (Email: azmemisge20@gmail.com)

\begin{abstract}
Objective: The study was aimed to investigate the determinants of time to drop out of treatment for TB patients.

Results: From 375 patients who started TB treatments about $24.8 \%$ dropout and $75.2 \%$ censored at the end of the study and the median survival time of TB patients were 199 days. The Log-rank results showed that marital status, HIV co infection, Diabetic mellitus, Cancer and Anemia cases had significant difference between the survival experience at $5 \%$ level of significance, whose different levels have an impact in the survival time of TB patients; whereas Sex, Phase of TB treatment, TB type, TB category, previous TB status, co-morbidity, and physical inactive had not significant difference between the survival experience at $5 \%$ level of significance. Finally, the result of Cox-proportion hazard model showed that, age, HIV co-infection and Anemia had a significant effect on tuberculosis patients during the study period.
\end{abstract}

Keywords: Tuberculosis; HIV co-infection; Cox-regression 


\section{Introduction}

Tuberculosis (TB) is a chronic infectious disease caused by Mycobacterium tuberculosis (MTB). TB typically affects the lungs (pulmonary tube) but, can affect other parts of the body as well (extra pulmonary tube). The Global tuberculosis report showed that TB now ranks above HIV as a leading cause of death worldwide [2]. An estimated incidence for the year 2015 of TB in Ethiopia was 191/100,000 population. In addition, as the national surveys on the burden of TB epidemic showed $31 \%-41 \%$ of TB patients are HIV positive [3]. The previous study shows that the prevalence of delayed presentation for HIV care among TB/HIV co-infected patients was $59.9 \%$. The study also shows tobacco non-users of TB/HIV co-infected participants were also $50 \%$ less likely to present late for HIV care compared to tobacco users. The relative odds of delayed presentation among Tb/HIV co-infected patients with ambulatory and bedridden functional status was higher than with working status [4]. The aim of this study is to identify risk factors that affect Survival Time to Drop out treatment among TB patients in case of BunoBedele and Illu Aba Bora zones, Oromia, Ethiopia.

\section{Methods:}

Secondary data was used from 375 TB patients of the selected health stations and hospitals at Buno-Bedele and Illu Aba Bora Zones. The response variable for this study was the survival time (Time to dropout the treatment among TB patients) measured in days and the covariates were gender of the patient, marital status, HIV co-infection, Phase of TB treatment, TB type, TB category, Previous TB history, HIV Co infection, Anemia and Physical inactivity. 


\section{Survival function}

In order to estimate the survival function, the estimator proposed by Kaplan and Meier takes into account for censoring by adjusting the number of subjects at risk,

$$
S_{k m}(t)=\prod_{i: t_{(i)} \leq t}\left[1-\frac{d_{i}}{n_{i}}\right]
$$

Where $t_{(i)}$ denote the distinct ordered times of Drop out and, $d_{i}$ and $n_{i}$ denote the number of events and the number of individuals still at risk at time $t_{i}$, respectively.

\section{Comparison of Survival Curves}

Kaplan-Meier method for estimating survival curves and the log-rank test for comparing two estimated survival curves are the most frequently used statistical tools in medical reports on survival data.

\section{Log-rank test}

The log rank test is a non-parametric test for comparing two or more independent survival curves. The log rank test statistic for comparing two groups is given by:

$$
Q=\frac{\left[\sum_{i=1}^{m} w_{i}\left(d_{1 i}-\widehat{e_{1 l}}\right)\right]^{2}}{\sum_{i=1}^{m} w_{i} \widehat{v_{1 l}}}
$$

Where: $m$ is the number of rank ordered event times, $d_{1 i}$ is the observed number of events in

group one at event time $t_{i}, \widehat{e_{1 \imath}}=\frac{n_{1 i}-d_{i}}{n_{i}}$ is the expected number of events corresponding to $d_{1 i}, n_{1 i}$ is the number of individuals at risk in group1 just prior to event time $t_{i}, n_{2 i}$ is the number of individuals at risk in group 2 just prior to event time $t_{i}$,

$$
\widehat{v_{1 \imath}}=\frac{n_{1 i} n_{2 i} d_{i}\left(n_{i}-d_{i}\right)}{n_{i}^{2}\left(n_{i}-1\right)}
$$


is the variance of the number of events $d_{1 i}$ at time $t_{i}, n_{i}$ and $d_{i}$ are the number of individuals at risk and number of vascular complication in both groups ( i.e., group 1 and group 2) just prior to event time $t_{i}$, respectively.

\section{The Cox ('Semi-Parametric') Proportional Hazards Model}

It is a survival analysis regression model, which describes the relation between the event incidence as expressed by the hazard function and a set of covariates. Mathematically, the Cox model is written as;

$$
h(t)=h_{0}(t) * \exp ^{\left(b_{1} x_{1}+b_{2} x_{2}+\ldots . .+b_{p} x_{p}\right)}
$$

where the hazard function $\mathrm{h}(\mathrm{t})$ is dependent on (or determined by) a set of $\mathrm{p}$ covariates $\left(\mathrm{x}_{1}, \mathrm{x}_{2}\right.$, $\left.\ldots, \mathrm{x}_{\mathrm{p}}\right)$, whose impact is measured by the size of the respective coefficients $\left(\mathrm{b}_{1}, \mathrm{~b}_{2}, . ., \mathrm{b}_{\mathrm{p}}\right)$. The term $\mathrm{h}_{0}(\mathrm{t})$ is called the baseline hazard, and is the value of the hazard if all the xi are equal to zero (the quantity exp (0) equals 1 ). The ' $\mathrm{t}$ ' in $\mathrm{h}(\mathrm{t})$ reminds us that the hazard may (and probably will) vary over time.

\section{RESULTS}

\section{Descriptive Statistics}

A total of $375 \mathrm{~TB}$ patients those treated during the study period were included in the study for which data for variables of interest are complete. Of all 375 TB patients 282(75.2\%) were censored or not experienced the event and 93(24.8\%) were patients dropout. The estimated median survival time of TB patient was $199(182.144,215.856)$ days. Of the total of 375 patients included, $184(49.3 \%)$ and 189(50.7\%) were Females and males respectively and their median of survival time was 198 and 220 days respectively. See table 1. 
Table 1: Demographic and health factors of categorical covariate by TB cases

\begin{tabular}{|c|c|c|c|c|c|c|}
\hline Covariate & Categories & $\begin{array}{l}\text { Number (\%) } \\
\text { Of study } \\
\text { Participants }\end{array}$ & $\begin{array}{l}\text { Number } \\
(\%) \\
\text { of dropout }\end{array}$ & $\begin{array}{l}\text { Number }(\%) \\
\text { of censored }\end{array}$ & Median & (Max, Min) \\
\hline \multirow[t]{2}{*}{ Sex } & Male & $189(50.4 \%)$ & $48(25.4 \%)$ & $141(74.6 \%)$ & 220 & {$[187.097,252.903]$} \\
\hline & Female & $186(49.6 \%)$ & $45(24.2 \%)$ & $141(75.8 \%)$ & 198 & {$[193.173,202.827]$} \\
\hline \multirow[t]{2}{*}{$\begin{array}{l}\text { Phase of } \\
\text { TB treatment }\end{array}$} & $\begin{array}{l}\text { Intensive } \\
\text { phase }\end{array}$ & $168(44.8 \%)$ & $42(25 \%)$ & $126(75 \%)$ & 199 & {$[179.138,218.862]$} \\
\hline & $\begin{array}{l}\text { Continuation } \\
\text { Phase }\end{array}$ & $207(55.2 \%)$ & $51(24.6 \%)$ & $156(75.4 \%)$ & 198 & {$[174.385,221.615]$} \\
\hline \multirow[t]{3}{*}{ TB type } & SPTB & $141(37.6 \%)$ & $38(27 \%)$ & $103(73 \%)$ & 212.867 & {$[200.300,225.433]$} \\
\hline & SNTB & $108(28.8 \%)$ & $27(25 \%)$ & $81(75 \%)$ & 202.388 & {$[183.708,221.067]$} \\
\hline & ETB & $126(33.6 \%)$ & $28(22.2 \%)$ & $98(77.8 \%)$ & 216.904 & [201.898, 231.909] \\
\hline \multirow[t]{4}{*}{ TB category } & New & $301(80.3 \%)$ & $76(25.2 \%)$ & $225(74.8 \%)$ & 212.239 & {$[201.526,222.952]$} \\
\hline & Relapse & $57(15.2 \%)$ & $15(26.3 \%)$ & $42(73.7 \%)$ & 221.500 & {$[219.421,223.579]$} \\
\hline & Failure & $9(2.4 \%)$ & $1(11.1 \%)$ & $8(88.9 \%)$ & 207.775 & {$[192.158,223.391]$} \\
\hline & Defaulter & $8(2.1 \%)$ & $1(12.5 \%)$ & $7(87.5 \%)$ & 229.326 & {$[215.546,243.107]$} \\
\hline \multirow{2}{*}{$\begin{array}{l}\text { Previous TB } \\
\text { history }\end{array}$} & No & $171(54.4 \%)$ & $49(24 \%)$ & $155(76 \%)$ & 168 & {$[158,168]$} \\
\hline & Yes & $171(45.6 \%)$ & $44(25.7 \%)$ & $127(74.3 \%)$ & 220 & {$[200.790,239.210]$} \\
\hline \multirow[t]{2}{*}{ HIV co infection } & Yes & $20(5.3 \%)$ & $86(24.2 \%)$ & $269(75.8 \%)$ & 201.607 & {$[190.394,212.820]$} \\
\hline & No & $355(94.7)$ & $7(35 \%)$ & $13(65 \%)$ & 168 & {$[162.878,173.122]$} \\
\hline \multirow[t]{2}{*}{ Co-morbidity } & No & $160(42.7 \%)$ & $41(25.6 \%)$ & $119(74.4 \%)$ & 198 & {$[180.909,215.091]$} \\
\hline & Yes & $215(57.3 \%)$ & $52(24.2 \%)$ & $163(75.8 \%)$ & 220 & {$[188.132,251.868]$} \\
\hline \multirow[t]{2}{*}{$\mathrm{DM}$} & Yes & $333(88.8 \%)$ & $9(21.4 \%)$ & $33(78.6 \%)$ & 216.381 & {$[201.086,231.677]$} \\
\hline & No & $42(11.2 \%)$ & $84(25.2 \%)$ & $249(74.8 \%)$ & 219.282 & {$[206.354,232.209]$} \\
\hline Cancer & Yes & $357(95.2 \%)$ & $7(38.9 \%)$ & $11(61.1 \%)$ & 220 & {$[202.25,237.745]$} \\
\hline
\end{tabular}




\begin{tabular}{|l|l|l|l|l|l|l|}
\hline & No & $18(4.8 \%)$ & $86(24.1 \%)$ & $271(75.9 \%)$ & 198 & $195.639,200.361$ \\
\hline \multirow{2}{*}{ Anemia } & Yes & $340(90.7 \%)$ & $12(34.3 \%)$ & $23(65.7 \%)$ & 219.975 & {$[204.397,235.553]$} \\
\cline { 2 - 7 } & No & $35(9.3 \%)$ & $81(23.8 \%)$ & $259(76.2 \%)$ & 209.427 & {$[195.069,223.785]$} \\
\hline Physical inactive & Yes & $4(1.1 \%)$ & $2(50 \%)$ & $2(50 \%)$ & 168 & {$[49.739,286.261]$} \\
\cline { 2 - 7 } & No & $371(98.9 \%)$ & $91(24.5 \%)$ & $280(75.5 \%)$ & 199 & {$[182.127,215.873]$} \\
\hline Total & 375 & $93(24.8 \%)$ & $282(75.2 \%)$ & 199 & {$[182.144,215.856]$} \\
\hline
\end{tabular}

The Kaplan- Meier survival curve Estimate of TB patients: Survival time and hazard rate of different variables

Figure 1 shows that overall Kaplan- Meier survival estimate decline as the survival time increase and it revealed that most of the dropout occurred in the first 150 days(during intensive phase) of treatment initiation, i.e. relatively, a large number of patients dropout at the earlier days of TB treatment initiation.

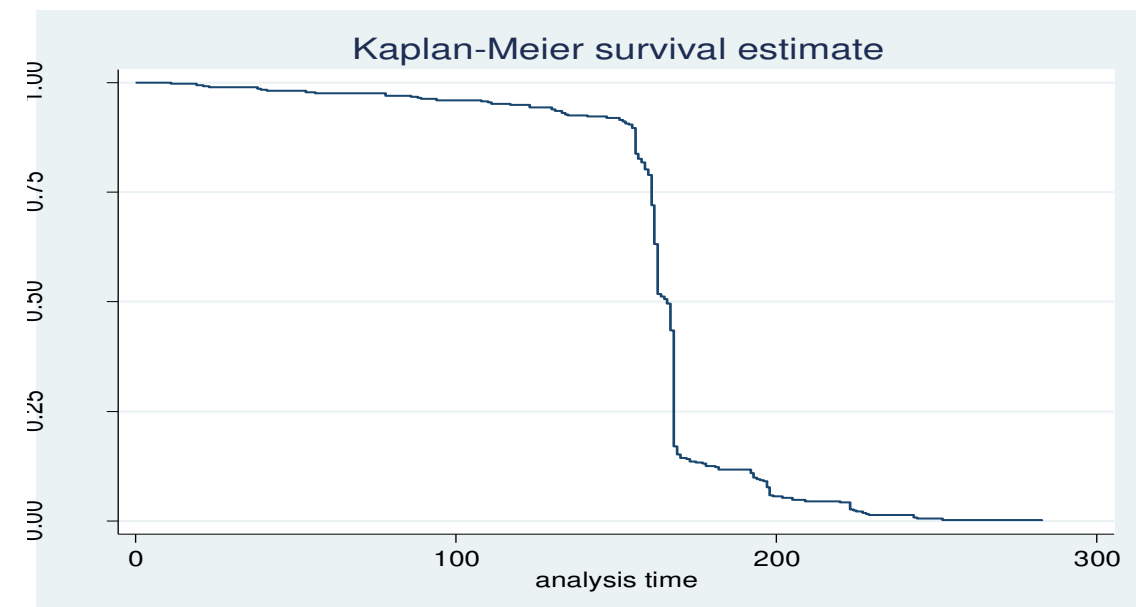

Figure 1: The Kaplan-Meier survival curve of the overall TB patients. 


\section{Log Rank Tests of Each Covariate and Comparison of survival time to dropout experience on TB patients}

Log-rank test and Kaplan-Meier survival estimates to look the significance of the difference in survival experience among different categories. The Log-rank results show that age, marital status, HIV co-infection, Diabetic mellitus, Cancer and Anemia cases had significant difference between the survival experience at 5\% level of significance, whose different levels have an impact in the survival time of TB patients; whereas Sex, Phase of TB treatment, TB type, TB category, previous TB status, co-morbidity, and physical inactive had not significant difference between the survival experience at $5 \%$ level of significance. The results of the Log-rank for the equality of survivor functions are presented in table 2.

Table 2: Comparison of Survival Experience of TB Patients Using Log-rank test

\begin{tabular}{|l|c|c|c|}
\hline Categorical covariates & \multirow{2}{*}{ Degree of } & \multicolumn{2}{|c|}{ Log -rank test } \\
\cline { 3 - 4 } & freedom & Chi-square & P -value \\
\hline Sex & 1 & 0.27 & 0.6034 \\
\hline Marital status & 3 & 16.56 & 0.0009 \\
\hline Phase of TB treatment & 1 & 0.27 & 0.6051 \\
\hline TB type & 2 & 2.32 & 0.3141 \\
\hline TB category & 3 & 1.01 & 0.7991 \\
\hline Previous TB status & 1 & 0.13 & 0.7170 \\
\hline Co-morbidity & 1 & 0.11 & 0.7414 \\
\hline HIV co infection & 1 & 5.38 & 0.0204 \\
\hline Diabetic mellitus & 1 & 19.42 & 0.0000 \\
\hline Cancer & 1 & 6.13 & 0.0133 \\
\hline
\end{tabular}




\begin{tabular}{|l|c|c|c|}
\hline Anemia & 1 & 17.87 & 0.0000 \\
\hline Physical inactive & 1 & 0.25 & 0.6176 \\
\hline
\end{tabular}

\section{Multivariable Cox Proportional Hazard Regression Analysis}

Multivariable Cox $\mathrm{PH}$ analysis (by using stepwise selection process) including all the potential risk factors that had a P-value of less than or equal 0.25 in single covariate Cox $\mathrm{PH}$ analysis. There are three covariates were significant at $5 \%$ level of significance. Hence; we have a final multivariate model which includes the three covariates namely: Age, HIV co-infection and Anemia are the risk factor for the dropout of TB patient or these variables significantly affects the survival of TB patients. See table S1.

\section{Test of the assumption of proportional hazard}

From the table S2, the overall global test for covariates was not satisfying the assumption of proportional hazard model, hence p-value is larger than 5\%. The global is no significant at $5 \%$ level of significance it means that the proportional hazard assumption is satisfied.

\section{Interpretation of results of Final Model of Cox regression model}

The interpretation from the results of the final model which consists of the main effects is based on the hazard ratios. Consequently, the interpretation of covariates that are included in the final proportion hazard model of TB dropout patients is as follows.

The hazard rate for patients whose Continuation Phase of TB treatment estimated was 1.088068 times with 95\% CI $[0.8826323,1.34132]$ meaning that patients in Continuation phase treatment had longer survival time than patients with intensive Phase treatment. The estimated hazard rate for patients with HIV Co-infection was estimated to be 1.75678 with $95 \%$ CI lies between 
[1.112293 and 2.774715]. P-value is less than 0.05, Meaning, patients with HIV Co-infection had 1.75678 times higher risk of TB treatment drop out than patients who didn't have HIV Coinfection.

\section{Parametric Regression Analysis}

For the data on TB patients, the parametric models were fitted. The common applicable criterion to select the model is the AIC statistic proposed by Akaikie (1983). From table S3, the Weibul regression model has the least AIC value which shows that the Weibul regression model well fitted to data TB patients.

\section{Discussion}

This research was conducted to identify predictors of drop out of treatment among TB patients. The Covariate included in the study were Sex, Age, Marital status, Anemia, Cancer, DM, Comorbidity, HIV Co-infection, Previous TB history, TB category, TB type, Physical inactive and Phase of TB treatment. The outcome of the interest was survival time of TB patient measured by days. The result of Cox regression analysis shows that age, HIV co-infection and Anemia were significant covariates of TB treatment drop out.

Of all 375 TB patients $282(75.2 \%)$ were censored or not experienced the event and 89 (24.8\%) were patients dropout. The estimated median survival time of TB patient was 199 (182.144, 215.856) days. TB patients with HIV Co-infection had 1.75678 times higher risks of TB treatment drop out than TB patients who didn't have HIV Co-infection. This finding is consistent with study conducted by $[5,6,7]$. It is important to highlight the high percentage $(63.7 \%)$ of individuals who did not undergo the anti-HIV test, even though the Ministry of Health's recommendation to perform the anti-HIV test in tuberculosis programs was established. It is 
estimated, in Brazil, that although the offer of anti-HIV test is approximately $70 \%$, only $50 \%$ of individuals have access to the result in due time; among these there is a prevalence of positivity of $15 \%$. In addition, tuberculosis is the major cause of death among people living with HIV, with a $20 \%$ death rate from co-infection. $[6,7]$.

TB patients who had anemia were 1.79 more likely to anti-TB treatment drop out than those TB patients who had not anemia. This finding supports the study conducted by SW Lee et al. [8]. They confirmed that anemia is a common hematological abnormality in patients with TB. Because TB-associated anemia is usually mild and resolves with anti-TB treatment, close observation is sufficient without other cause of the anemia.

\section{Conclusions}

The main aim of this study was investigating the determinants of time to drop out of treatment for TB patients. Secondary data was used from 375 TB patients of the selected health stations and hospitals at Buno-Bedele and Illu Aba Bora Zones. The response variable for this study was the survival time (Time to dropout the treatment among TB patients) measured in days and the covariates were gender of the patient, marital status, HIV co-infection, Phase of TB treatment, TB type, TB category, Previous TB history, HIV Co infection, Anemia and Physical inactivity. Descriptive statistics, Kaplan-Meier Estimation method, Semi-parametric survival models and parametric survival models were used for the analysis of time to TB treatment dropout dataset. The result of this study revealed that among 375 patients who started TB treatments about $24.8 \%$ dropout and $75.2 \%$ censored at the end of the study and the median survival time of TB patients were 199 days. The Log-rank results showed that marital status, HIV co infection, Diabetic mellitus, Cancer and Anemia cases had significant difference between the survival experience at $5 \%$ level of significance, whose different levels have an impact in the survival time of TB 
patients; whereas Sex, Phase of TB treatment, TB type, TB category, previous TB status, comorbidity, and physical inactive had not significant difference between the survival experience at $5 \%$ level of significance. Finally, the result of Cox-proportion hazard model showed that, age, HIV co-infection and Anemia had a significant effect on tuberculosis patients during the study period. It is suggested that for reducing TB treatment drop out, due emphasis should be given in improving the knowledge and practice of TB patient's for appropriate use of treatment till the patient is cure.

\section{Abbreviations}

AIC: Akaike Information Criterion; DM: Diabetic Mellitus; HIV: Human Immunodeficiency Viruses; TB: Tuberculosis; WHO: World Health Organization.

\section{Declarations}

Funding: No funding was obtained for this study.

Ethics approval and consent to participate: Not applicable

Consent for publication: Not applicable

Competing of interests: The authors declare that they have no competing interests

Availability of data and materials: We can provide the dataset that has been used to do this study up on reasonable request.

Authors' contribution: All authors contributed equally to the study. WE conceived the idea, DG; AM contributed in the design analyses and interpretation, WE the corresponding author drafted the manuscript. All authors read and approved the final manuscript. 
Acknowledgments: The authors gratefully acknowledge all health sectors in Illu- Ababora and Buno-Bedelle Zones, Oromia Regional State, Ethiopia for allowing us to use the data.

\section{Author details:}

${ }^{1,2,3}$ Department of Statistics, Faculty of Natural and Computational Sciences, Mettu University, Mettu, Ethiopia.

\section{REFERENCE}

[1]. Akaike.1983.Information Measures and Model Selection, Bulletin of the International Statistical Institute, 50, 277-290. Accessed on July 03, 2014.

[2]. Hosmer DW, Lemeshow S. Regression modeling of time-to-event data. Wiley, NewYork. 1999.

[3]. World Health Organization (WHO). 2016.. "Global Tuberculosis Report: global actions and investments fall far short of those needed to end the global TB”, Geneva

[4]. Hailay Gesesew, Birtukan Tsehaineh, Desalegn Massa, Amanuel Tesfay, Hafte Kahsay and Lillian Mwanri (2016). The prevalence and associated factors for delayed presentation for HIV care among tuberculosis/HIV co-infected patients in Southwest Ethiopia: a retrospective observational cohort. DOI 10.1186/s40249-016-0193-y

[5]. Garrido MdS, Penna ML, Perez-Porcuna TM, Souza ABd, Marreiro LdS, et al. (2012) Factors Associated with Tuberculosis Treatment Default in an Endemic Area of the Brazilian Amazon: A Case Control-Study. PLoS ONE 7(6): e39134. doi:10.1371/journal.pone.0039134 
[6]. PF Silva, GS Moura, AJM Caldas, (2014). Factors associated with pulmonary TB treatment dropout in Maranhão State, Brazil, from 2001 to 2010. https://doi.org/10.1590/0102-311X00124513

[7]. Department of Epidemiological Surveillance, Secretariat of Health Surveillance, Ministry of Health. Manual of recommendations for tuberculosis control in Brazil. Brasília: Ministry of Health; 2011.

[8]. Sei Won Lee, Young Ae Kang, Young Soon Yoon, Sang-Won Um, Sang Min Lee, ChulGyu Yoo, Young Whan Kim, Sung Koo Han, Young-Soo Shim, and Jae-Joon Yim. The Prevalence and Evolution of Anemia Associated with Tuberculosis. J Korean Med Sci. 2006 Dec; 21(6): 1028-1032doi: 10.3346/jkms.2006.21.6.1028 
Figures

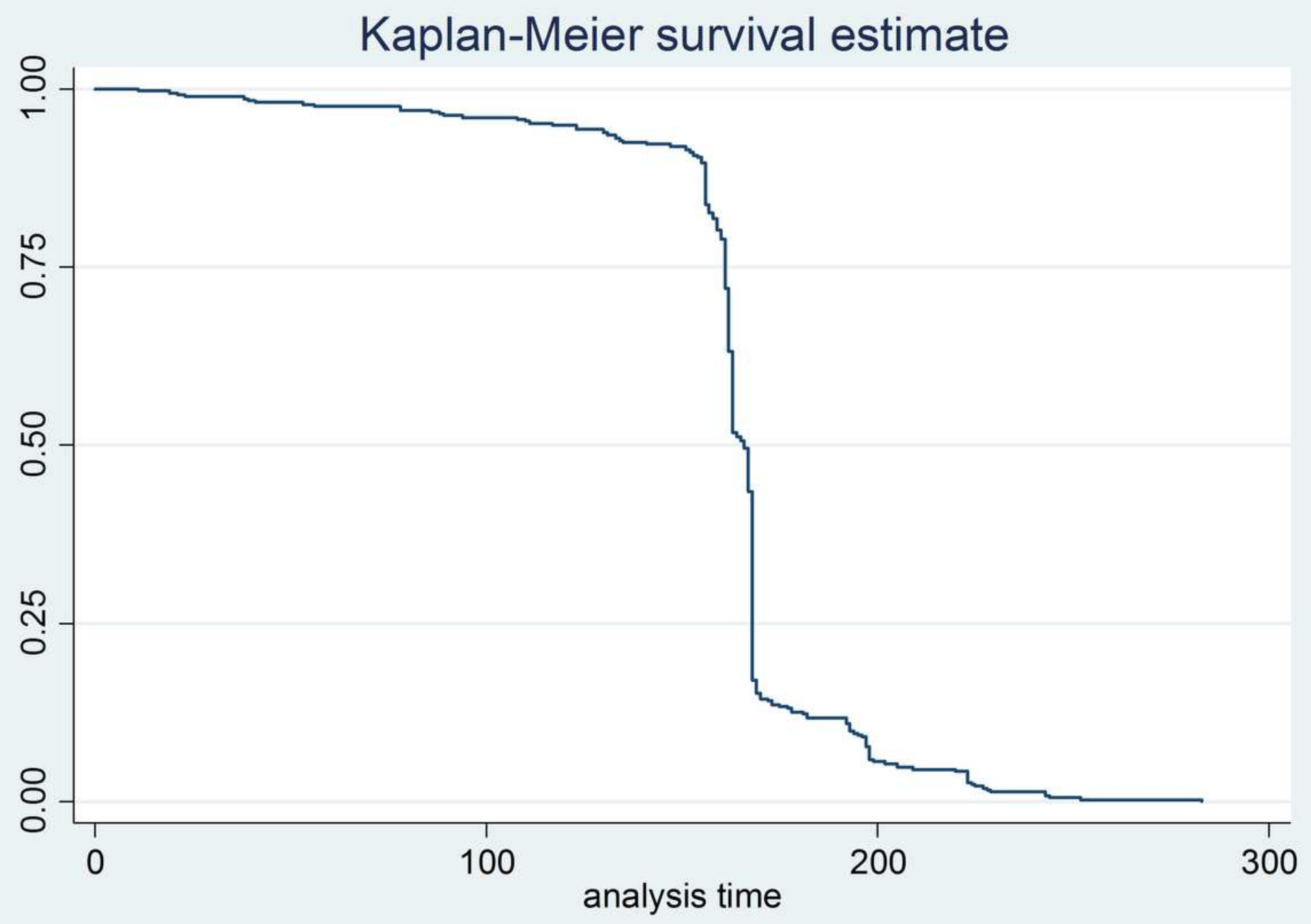

Figure 1

The Kaplan-Meier survival curve of the overall TB patients.

\section{Supplementary Files}

This is a list of supplementary files associated with this preprint. Click to download.

- SupplementarymaterialsTB.docx 